\title{
How to Cope with Big Data in Functional Analysis of the Esophagus
}

\author{
Alissa Jell $^{\mathrm{a}}$ Christina Kuttler $^{\mathrm{b}} \quad$ Daniel Ostler $^{\mathrm{c}} \quad$ Norbert Hüser $^{\mathrm{a}}$ \\ ${ }^{a}$ Department of Surgery, Medical Faculty, University Hospital Klinikum rechts der Isar, Technical University of \\ Munich, Munich, Germany; ${ }^{\mathrm{b}} \mathrm{M6}$ - Mathematical Modelling, Mathematical Faculty, Technical University of Munich, \\ Munich, Germany; ${ }^{c}$ Minimally Invasive Interdisciplinary Therapeutical Intervention (MITI) Research Group, Medical \\ Faculty, Technical University of Munich, Munich, Germany
}

\section{Keywords}

Long-term high-resolution manometry · Esophagus · Big data - Artificial intelligence - Automated swallow detection . Classification

\begin{abstract}
Introduction: Esophageal motility disorders have a severe impact on patients' quality of life. While high-resolution manometry (HRM) is the gold standard in the diagnosis of esophageal motility disorders, intermittently occurring muscular deficiencies often remain undiscovered if they do not lead to an intense level of discomfort or cause suffering in patients. Ambulatory long-term HRM allows us to study the circadian (dys)function of the esophagus in a unique way. With the prolonged examination period of $24 \mathrm{~h}$, however, there is an immense increase in data which requires personnel and time for evaluation not available in clinical routine. Artificial intelligence ( $\mathrm{Al}$ ) might contribute here by performing an autonomous analysis. Methods: On the basis of 40 previously performed and manually tagged long-term HRM in patients with suspected temporary esophageal motility disorders, we implemented a supervised machine learning algorithm for automated swallow detection and classification. Results: For a set of $24 \mathrm{~h}$ of long-term HRM by means of this algorithm, the evaluation time could be reduced from 3 days to a core evaluation time of $11 \mathrm{~min}$ for automated swallow detection and clustering plus an additional 10-20 min of evaluation time, depending on the complexity and diversity of motility disorders in the examined patient. In $12.5 \%$ of patients with suggested esophageal motility disorders, Al-en-
\end{abstract}

abled long-term HRM was able to reveal new and relevant findings for subsequent therapy. Conclusion: This new approach paves the way to the clinical use of long-term HRM in patients with temporary esophageal motility disorders and might serve as an ideal and clinically relevant application of Al.

() 2020 S. Karger AG, Basel

\section{Introduction}

Esophageal motility disorders lead to highly heterogeneous symptoms ranging from mild, intermittently occurring forms to noncardiac chest pain and severe forms of dysphagia that decisively impact the patient's quality of life. Dysphagia especially occurs in elderly people; oropharyngeal dysphagia is present in up to $33 \%$ of patients above the age of 65 years $[1,2]$, while esophageal dysphagia occurs in 1 of 6 adults [3] with a severe risk of isolation if they are no longer able to eat in public, even leading to malnutrition. Thus, the World Health Organization (WHO) recognized swallowing disorders as a "medical disability associated with increased morbidity, mortality and costs of care" [4].

The gold standard in the diagnosis of esophageal motility disorders, after having ruled out malignancy by endoscopy, is esophageal high-resolution manometry (HRM) [5]. HRM is usually performed in a very limited period of time and in accordance with a well-defined swallow protocol (typically around $30 \mathrm{~min}$ ) under laboratory conditions. The examination is evaluated afterwards 


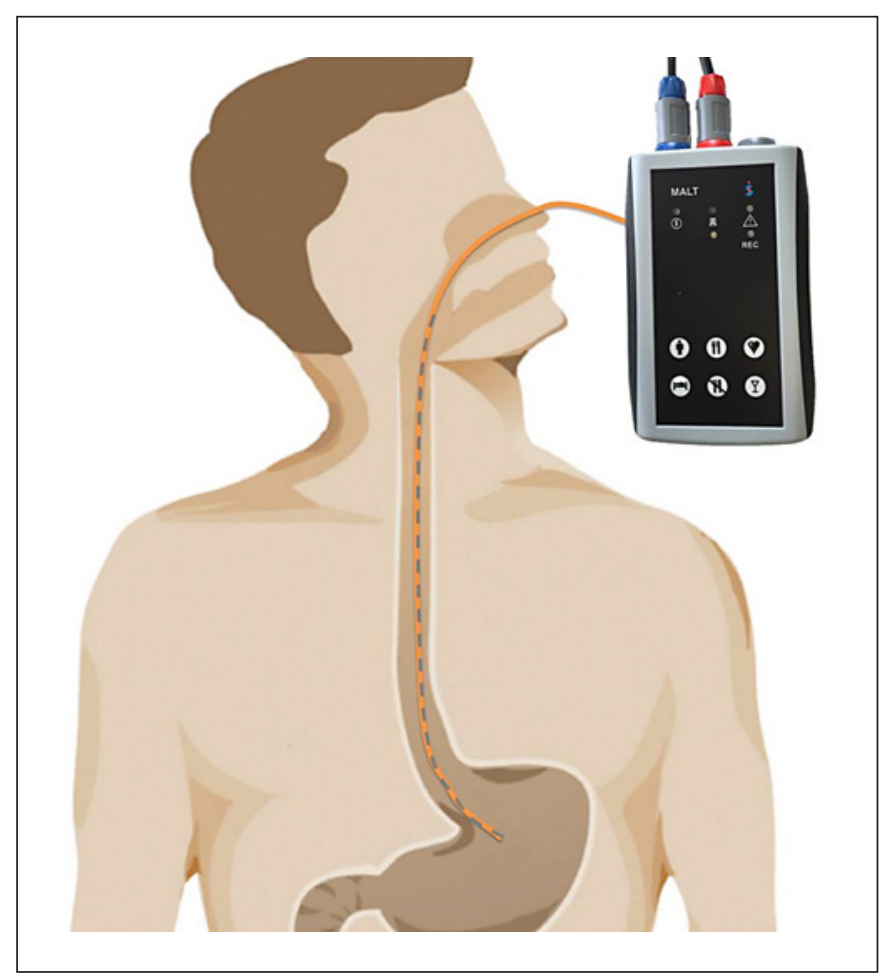

Fig. 1. Illustration of a long-term HRM with a 10-Fr HRM catheter (a) connected to the MALT ${ }^{\mathrm{TM}}$ recording device (b).

according to Chicago Classification (CC) criteria [6]. Patients with only intermittent symptoms that are missed during HRM are often repeatedly referred to specialists or sometimes treated as simulants. Of note, CC is only applicable if the predefined criteria are met during the standardized examination (e.g., $45^{\circ}$ elevated body position, defined water boli of $5 \mathrm{ml}$ ). There exists only very little knowledge on how manometric measurements vary over a longer period of time, e.g., over $24 \mathrm{~h}$. Moreover, approximately one third of patients examined by HRM have findings not covered by CC [7].

To evaluate so far unrecognized pathologies and to improve the current measure for the identification of motility disorders, we prolonged HRM to $24 \mathrm{~h}$. As we aimed for maximum representativeness of the patient's routine, investigations were performed in an outpatient setting [8]. Due to the extended scanning time, this leads to a substantial increase in data and work when evaluating long-term HRM. This increased work is mainly derived from the fact that, up to now, the evaluation has been carried out manually. Indeed, specialized physicians are blocked for about 3 working days to assess a single 24-h long-term HRM examination. Comprehensively, and due to the huge amount of data, these examinations are neither easy to analyze by hand nor applicable in clinical routine and are performed, if at all, only in the context of scientific research. Our previous work showed that by long-term HRM, in about every eighth patient with a clinically apparent but so far unrecognized esophageal motility disorder, new and relevant findings could be revealed [8]. In order to being able to use this diagnostic possibility in clinical practice in the future, we developed an artificial intelligence (AI)-based algorithm for automated swallow detection and classification. The algorithm was intended to cope with big data of long-term HRM in day-to-day practice.

\section{Materials and Methods}

\section{Long-Term HRM: Technique and Data}

For long-term HRM we used a common solid-state manometry catheter (Laborie Medical Technologies, USA; Fig. 1a) with a 10-Fr diameter and 36 circular pressure sensors distributed along the probe at $1-\mathrm{cm}$ equal distances. After performing conventional esophageal motility analysis according to the well-established examination protocol for CC, the probe was connected to an ambulatory recording device (MALT ${ }^{\mathrm{TM}}$; Standard Instruments $\mathrm{GmbH}$, Germany; Fig. 1b) to continue with long-term HRM observation. With an internal storage capacity of $1 \mathrm{~GB}$ the ambulatory recording unit allowed for high-quality, state-of-the-art, high-resolution examinations over at least $26 \mathrm{~h}$ at a sampling frequency of $50 \mathrm{~Hz}$.

For training and evaluation, 40 long-term HRM of patients with suspected temporary motility disorders were used. All patients were diagnosed at the surgical gastrointestinal function diagnostic laboratory of the Klinikum rechts der Isar (Technical University of Munich, Germany) and underwent medically indicated long-term HRM beforehand. As the gold standard for further analyses, all long-term HRM were manually tagged accurately by medical experts with at least 10 years of experience in esophageal manometry using ViMeDat ${ }^{\mathrm{TM}}$ software (Standard Instruments).

The final long-term HRM datasets (CSV files) each included 36 pressure channels at a sampling rate of $50 \mathrm{~Hz}$ as well as patient- and examination-specific data (e.g., demographic data, body position, and eating or resting times) and a list of manually referenced relevant anatomical sites (e.g., upper and lower esophageal sphincters) and all swallows with marked beginnings, endings, transitions zones, proximal and distal contractile front velocity, and areas of relaxation of the esophageal sphincters. The information summed up to a dataset of about 36 (pressure channels) $\times 4,320,000$ $(50 \mathrm{~Hz}$ over $24 \mathrm{~h})+900$ (average acts of swallowing per day) $\times 7$ (markers) and hence 155 million data points per patient and measurement. The raw manometric data together with the manual annotations resulted in data sets as big as $1.5 \mathrm{~GB}$.

Training and Evaluation of the System

For training of our machine learning algorithm, 15 long-term HRM were used, while 25 long-term HRM were kept for later cross-validation.

All data sets were converted into matrices of $36 \times n$ (number of manually tagged swallows) in size. As human measurements in general and HRM measurements in particular are highly sensitive to background noises (e.g., breathing, heartbeat, speaking, and coughing) all data were first filtered and aggregated at the variance level. Explicitly, pressure recordings of each patient were transformed and standardized to the mean pressure with an indication of the specific SD.

Esophageal motility disorders are typically associated with swallowing but can also be present independently and in between swallows. To also identify these unassociated dysfunctions of esophageal motility, we increased the detection rate for swallows, albeit 


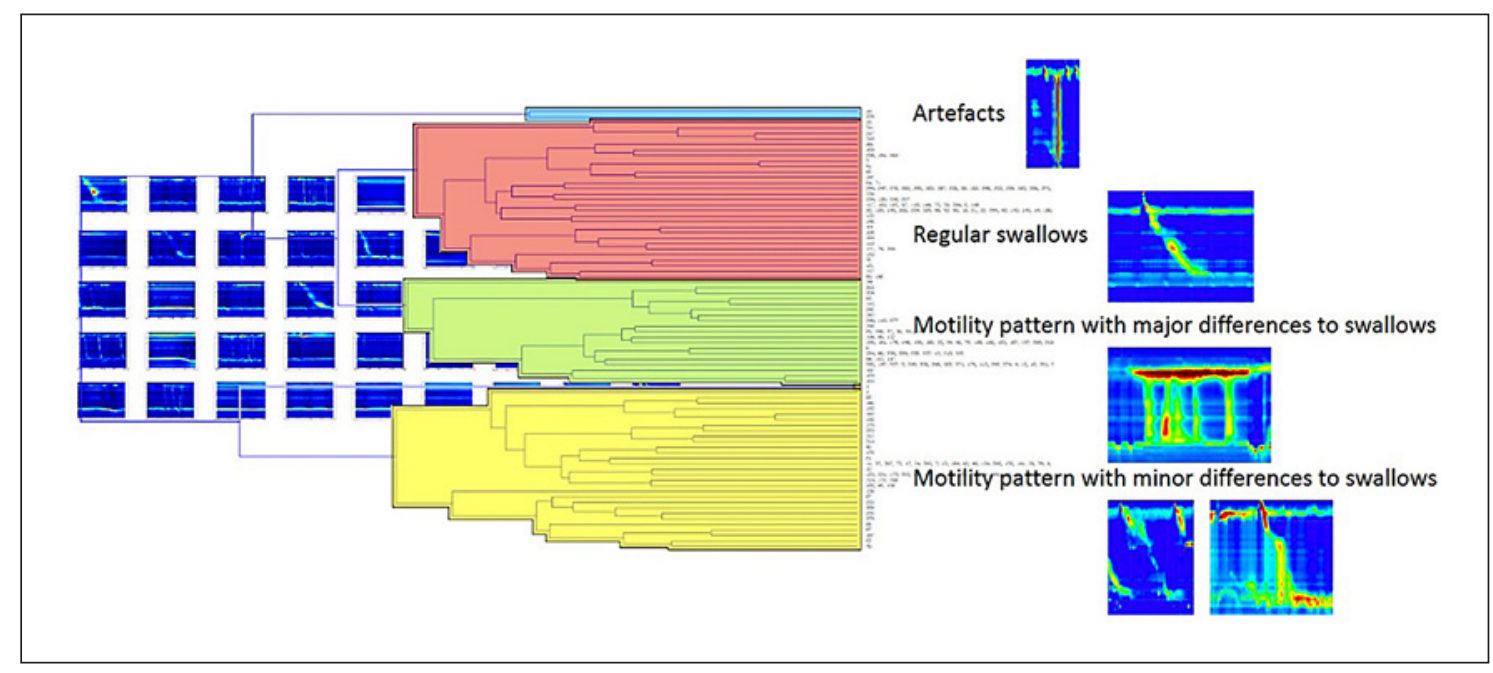

Fig. 2. Exemplary deducted images of swallows and esophageal motility by automated swallow detection clustered into the following 4 groups: regular swallow (a), major (b) and minor (c) motility disorders, and artefacts (d).

with the risk of obtaining false-positive results. By using a weighted learning algorithm and according to the empirical risk minimization, we were able to minimize false-positive assumptions.

Sole automated swallow detection is a first big step toward automated analysis of long-term HRM measurements but it is not enough to be able to evaluate long-term HRM in clinical routine. In addition, a classification and clustering scheme was therefore implemented in our algorithm. First, the well-established colorcoded manometric plots for HRM were deducted for each detected swallow in image sizes of $640 \times 480$ square pixels. The relaxation of the upper esophageal sphincter was set as starting at frame 50 (hence after $1 \mathrm{~s}$ ) to adequately depict predeglutitive pressurizations and motility changes, especially in the hypopharyngeal area. The structural similarity index in $4 \times 4$ patterns was then calculated and all captured images were classified according to the current CC criteria. Exemplarily, Figure 2 shows the distribution and classification of esophageal motility patterns during long-term HRM.

\section{Results}

On the basis of 40 previously performed and precisely (manually) tagged long-term HRM in symptomatic patients with intermittent esophageal motility disorders, we developed and implemented a supervised machine learning algorithm for automated swallow detection and classification.

Swallow detection alone will reduce the evaluation time by $40 \%$, and hence from 3 working days will be reduced to about $15 \mathrm{~h}$; however, this is still too long for clinical use. Therefore, and in addition to swallow recognition, an automated classification on the basis of the well-established CC criteria for motility disorders was implemented. By this, the evaluation time for a 24-h longterm HRM could be further reduced to a mean evaluation time of $11 \mathrm{~min}$ (for automated swallow detection and clustering). An additional 10-20 min of evaluation time might be necessary depending on the complexity and diversity of motility disorders in the examined patient. However, with the availability of greater computing power, a further reduction in the examination time may be possible and could pave the way to further broad clinical applications.

With the combination of a weighted learning algorithm and empirical risk minimization, best results were achieved with a weighted risk, i.e., $R$, of 0.149 with an error, i.e., $e$, of only $2.3 \%$ for precisely detecting both swallows and nonswallows. Thus, with a specificity of $83.2 \%$ and a sensitivity of $89.7 \%$, the optimal weighted risk $(R)$ was minimized so that only few false-positive motility patterns were detected in favor of increased swallow detection. Among these, merely motility patterns were found which were linked to abdominal-thoracic background noises with fast changes in pressure levels (e.g., coughing, hiccups) similar to those well known in the hypopharyngeal and upper esophageal sphincter area during swallow-reflecting relaxation. Despite their general similarity to conventional swallowing patterns, those pressurizations could be filtered on the basis of their short duration, which makes automated swallow detection and clustering even more reliable and effective.

By the application of an AI algorithm, the analysis of long-term HRM is for the first time achievable in a clinically acceptable time span with a high accuracy of $97.7 \%$.

\section{Conclusion}

To date, AI applications have been intensively discussed and applied to various fields in medicine, but, admittedly, useful applications that really provide true sup- 
port are lacking. One relevant aspect might be that most medical data are still registered in an analog form, which requires translation to digital data sets before AI-based analysis becomes feasible. Sometimes, the complexity of information hinders an autonomous compilation or evaluation and interpretation is highly human dependent. In contrast, image data (e.g., radiologic data, skin photographs) have been shown to be ideal candidates for this methodology and were set first in line as good indications for AI in medicine [9]. However, as a patient only provides a single set of images at a given time, the high complexity and variance of findings is inherent within this data, so building up a reliable ground truth is demanding and often requires huge data sets.

In our opinion, long-term HRM could be ideal as it registers information in images that are highly standardized and can already be classified in a much defined manner according to the CC. Due to long-term measurements over $24 \mathrm{~h}$ for each patient, a large amount of comparable but circadian-variable information is collected, which allows us to easily and effectively form the necessary ground truth. Analysis of these large data sets, on the other hand, is time demanding. AI for the analysis of HRM could be an outstanding application of this attractive technology and was found to be effective even with a small cohort size.

An accuracy of almost $98 \%$ was achieved by supervised machine learning algorithms on apparently small data (40 data sets) with, nevertheless, a large data set with a high resolution (155 million measuring points per data set). Thanks to AI-based analysis, we have demonstrated for the first time that long-term HRM can be made applicable for regular clinical operation outside of studies. With this autonomous analytic approach, we pave the way for a broad application which will serve as a basis for a better understanding of circadian esophageal (mal) function.

In contrast to AI, where algorithms do not ponder the available information to come to a decision, physicians intuitively use their perception and expertise for data analysis, with the latter being often required in the healthcare environment. Because of the complex heterogeneity of data and the nontransparent interdependence of information in medicine, the necessary interpretation with intuition and weighting so far hinders the widespread use of AI in medicine. If the application is well chosen, however, $\mathrm{AI}$ and in particular smart intelligence applications will perfectly support doctors in their daily work and help to diagnose diseases more safely and efficiently and personalize medications and treatments.

In our opinion, the use of smart AI in this setting is both justifiable and of direct benefit to the physician, as it leads to a significant reduction in the workload. Further work in this area is desirable and highly warranted.

\section{Acknowledgement}

Important development of the automated swallow detection algorithm part was made by the Case Studies Students Team Season 2019 under the leadership of Prof. C. Kuttler.

\section{Statement of Ethics}

This study was conducted with the approval of the institutional ethical approval committee under approval number 135/19 S. All investigations were medically indicated and written informed consent was obtained from all of the patients.

\section{Conflict of Interest Statement}

The authors have no conflict of interests to declare.

\section{Funding Sources}

No funding was received for this work.

\section{Author Contributions}

A.J. and N.H. did the data acquisition. A.J. performed the evaluation and wrote this paper. A.J., C.K., and D.O. performed the data analysis. D.O. and N.H. reviewed this paper.

\section{References}

1 Perry L, Love CP. Screening for dysphagia and aspiration in acute stroke: a systematic review. Dysphagia. 2001;16(1):7-18.

2 Yang EJ, Kim MH, Lim JY, Paik NJ. Oropharyngeal Dysphagia in a community-based elderly cohort: the korean longitudinal study on health and aging. J Korean Med Sci. 2013 Oct; 28(10):1534-9.

3 Adkins C, Takakura W, Spiegel BM, Lu M, Vera-Llonch M, Williams J, et al. Prevalence and Characteristics of Dysphagia Based on a Population-Based Survey. Clin Gastroenterol Hepatol. 2020 Aug;18(9):1970-1979.e2.
4 International classification of functioning, disability, and health: ICF. Geneva: World Health Organization; 2001.

5 Yadlapati R. High resolution manometry vs conventional line tracing for esophageal motility disorders. Gastroenterol Hepatol (NY). 2017 Mar; 13(3):176-8.

6 Kahrilas PJ, Bredenoord AJ, Fox M, Gyawali CP, Roman S, Smout AJ, et al.; International High Resolution Manometry Working Group. The Chicago classification of esophageal motility disorders, v3.0. Neurogastroenterol Motil. 2015 Feb;27(2):160-74.
7 Wang YT, Yazaki E, Sifrim D. High-resolution manometry: esophageal disorders not addressed by the "Chicago Classification". J Neurogastroenterol Motil. 2012 Oct;18(4): 365-72.

8 Jell A, Wilhelm D, Ostler D, Feussner H, Hüser N. Longterm HR manometry of the esophagus: first findings in clinical use. J Gastroenterol. 2016 Sep;42(9):1069-75.

9 Hosny A, Parmar C, Quackenbush J, Schwartz LH, Aerts HJ. Artificial intelligence in radiology. Nat Rev Cancer. 2018 Aug;18(8):500-10. 